\title{
Bacteriological findings and antimicrobial resistance in odontogenic and non-odontogenic chronic maxillary sinusitis
}

\begin{abstract}
Correspondence
Annamaria Speciale special@unict.it
\end{abstract}

Received 15 February 2011

Accepted 15 April 2011

\author{
Salvatore Puglisi, ${ }^{1}$ Salvatore Privitera, ${ }^{1}$ Luigi Maiolino, ${ }^{2}$ Agostino Serra, ${ }^{2}$ \\ Matteo Garotta, ${ }^{3}$ Giovanna Blandino ${ }^{1}$ and Annamaria Speciale ${ }^{1}$ \\ ${ }^{1}$ Department of Bio-medical Sciences, Section of Microbiology, University of Catania, Catania, Italy \\ ${ }^{2}$ Department of Medicine and Surgery, Section of Otolaryngology, University of Catania, Catania, \\ Italy \\ ${ }^{3}$ Department of Otorhinolaryngology, Foundation IRCCS Policlinico San Matteo, University of Pavia, \\ Pavia, Italy
}

\begin{abstract}
The main objectives of this study were to estimate the frequency of chronic maxillary sinusitis of dental origin, and to evaluate the microbiology of odontogenic and non-odontogenic chronic maxillary sinusitis. Aspirates from 59 patients with chronic maxillary sinusitis (47 non-odontogenic, 12 odontogenic), collected during a 3-year period, were microbiologically processed for aerobic and anaerobic bacteria. Moreover, antimicrobial susceptibility was evaluated in the isolated bacteria. In this study, $20 \%$ of chronic maxillary sinusitis cases were associated with a dental origin, and sinus lift procedures were the main aetiological factor. Our microbiological findings showed that all specimens from chronic maxillary sinusitis were polymicrobial. Sixty aerobes and 75 anaerobes were recovered from the 47 cases of non-odontogenic sinusitis ( 2.9 bacteria per specimen); 15 aerobes and 25 anaerobes were isolated from the 12 patients with odontogenic sinusitis (3.3 bacteria per specimen). The predominant aerobes were Staphylococcus aureus (27) and Streptococcus pneumoniae (16), while the more frequent anaerobes were

Peptostreptococcus species (31) and Prevotella species (30). Haemophilus influenzae and Moraxella catarrhalis were absent in sinusitis associated with a dental origin. Overall, $22 \%$ of Staphylococcus aureus isolates were oxacillin-resistant, and $75 \%$ of Streptococcus pneumoniae isolates were penicillin-resistant and/or erythromycin-resistant; $21 \%$ of anaerobic Gram-positive bacteria were penicillin-resistant, and $44 \%$ of anaerobic Gram-negative bacteria were $\beta$ lactamase-positive. Vancomycin and quinopristin-dalfopristin had the highest in vitro activity against Staphylococcus aureus and Streptococcus species, respectively; amoxicillin-clavulanate and cefotaxime showed the highest in vitro activity against aerobic Gram-negative bacteria; and moxifloxacin, metronidazole and clindamycin were the most active against anaerobic bacteria.
\end{abstract}

\section{INTRODUCTION}

Maxillary sinusitis is defined as symptomatic inflammation of the paranasal maxillary sinuses, and is further classified as chronic when its duration exceeds 12 weeks, with or without acute exacerbations. Chronic maxillary sinusitis is one of the most common health-care problems in the world (Benninger et al., 2000). The sinusitis is primarily rhinogenous, but because of the anatomical juxtaposition of the teeth and the maxillary sinus sometimes it can be associated with a predisposing odontogenic condition (Kretzschmar \& Kretzschmar, 2003).

Sinusitis arising from dental disease differs in its microbiology and management from sinusitis due to other

Abbreviation: CLSI, Clinical and Laboratory Standards Institute. causes. Many studies have examined the bacterial pathogens associated with chronic sinusitis, but the great majority of them included only a small, or indeterminate, number of patients with odontogenic infection (Kremer et al., 2001; Finegold et al., 2002; Aral et al., 2003; Niederfuhr et al., 2009), or did not test the antimicrobial susceptibility of these bacteria (Brook, 2005). While it is reported that the sinusitis of dental origin is polymicrobial and caused by bacteria from the oral cavity and from the upper airways (Mehra \& Jeong, 2009), some aspects, such as the role of anaerobic bacteria in this infection, are not well studied and would need to be elucidated.

The objectives of this study were to detect aerobic and anaerobic bacteria involved in the aetiopathogenesis of chronic maxillary sinusitis, secondary or not to odontogenic 
predisposing factors, to evaluate the antimicrobial susceptibility of these bacteria, and to investigate which odontogenic causes can lead to chronic maxillary sinusitis.

\section{METHODS}

Patients. A total of 59 patients (31 male and 28 female; 45-82 years old; mean age 57 years) with a history of chronic maxillary sinusitis, who were referred to an ear, nose and throat surgeon at the 'Azienda Policlinico-Universitaria Policlinico-Vittorio Emanuele' for endoscopic sinus surgery, were studied between October 2007 and September 2010.

The diagnosis of chronic sinusitis was based on a thorough medical history assessment and physical examination, and on radiological findings, including a paranasal sinus CT scan. Clinical examination of the teeth and adjacent tissues was also performed on all patients. Chronic maxillary sinusitis was considered to be of rhinogenous origin when the dental examination was normal. When the examination of dental status showed clinical disease of the periodontal tissues, or of endodontically involved teeth, dental radiological examination (orthopantomography) was also performed. Chronic maxillary sinusitis was considered to be of dental origin when the dental examination and the radiograph showed a close connection between the diseased maxillary sinus mucosa and an inflammatory periodontal or periapical lesion.

All patients were treated with functional endoscopic sinus surgery.

This study conformed to the guidelines of the Ethical Committee of the University of Catania (Italy). All patients gave informed consent to the work.

Specimen collection and microbiological procedures. Specimens were aseptically obtained from sinus during surgery by antral puncture of the canine fossa or the lateral nasal cavity wall, before the patient had undergone perioperative antibiotic prophylaxis. Specimens were transported in a sealed syringe or in an anaerobic transport vial (BBL Port-A-Cul Vial) to the microbiology laboratory within $30 \mathrm{~min}$ of collection.

Microbiological assessments were started on the arrival of the sample at the laboratory. All samples were inoculated on Columbia agar with $5 \%$ sheep blood (BD Becton Dickinson), Haemophilus Test Medium (HTM) agar (BD Becton Dickinson) and Brucella blood agar with haemin and vitamin $\mathrm{K}_{1}$ (BD Becton Dickinson). Columbia blood and HTM agar plates were incubated under aerobic conditions and in an atmosphere of $10 \% \mathrm{CO}_{2}$ at $35{ }^{\circ} \mathrm{C}$ for $24-48 \mathrm{~h}$. Supplemented Brucella agar plates were incubated anaerobically in GasPak 100 Jars (BD Becton Dickinson) at $35{ }^{\circ} \mathrm{C}$ for $72 \mathrm{~h}$; the incubation was continued for at least 7 days, even in the absence of bacterial growth (Blandino et al., 2007). From each bacterial plate, representative colonies of each morphological type were subcultured on selective media (Murray et al., 2007). Identification of bacteria was made by colony and cellular morphology, staining characteristics and commercially available microbiological identification systems, such as API 20 Strep, API 20 E, API 20 NE, API NH, API 20 A, Rapid ID 32 A and API ZYM (bioMérieux) (Summanen et al., 1993; Blandino et al., 2007; Murray et al., 2007).

Antibiotics. We studied the antimicrobial susceptibility of aerobic Gram-positive bacteria to the following antibiotics: penicillin, oxacillin (only for staphylococci), vancomycin (only for staphylococci), clindamycin, erythromycin, quinopristin-dalfopristin and moxifloxacin (except for $\beta$-haemolytic group streptococci). We studied the antimicrobial susceptibility of aerobic Gram-negative bacteria to the following antibiotics: ampicillin, amoxicillin-clavulanate, cefotaxime, ceftazidime (only for members of the Enterobacteriaceae), moxifloxacin and trimethoprim-sulfamethoxazole (co-trimoxazole). We tested the antimicrobial susceptibility of anaerobic bacteria to the following antibiotics: penicillin, clindamycin, metronidazole and moxifloxacin.

Antimicrobial susceptibility and $\boldsymbol{\beta}$-lactamase testing. The antimicrobial susceptibility of aerobic bacteria was determined by evaluating the MIC by the broth microdilution method, as recommended by the Clinical and Laboratory Standards Institute (CLSI) (CLSI, 2009). The strains were defined as susceptible or resistant to the tested antibiotics according to the MIC breakpoints suggested by the CLSI (CLSI, 2010a, b). For moxifloxacin against members of the Enterobacteriaceae and Moraxella catarrhalis, since the CLSI has not established breakpoints, the definitions were based on the European Committee on Antimicrobial Susceptibility Testing breakpoint tables for interpretation of MIC (http://www.eucast.org/fileadmin/src/media/ PDFs/EUCAST_files/Disk_test_documents/EUCAST_breakpoints_v1. 1.xls). Staphylococcus aureus ATCC 29213, Streptococcus pneumoniae ATCC 49619 and Haemophilus influenzae ATCC 49247 were used for quality-control purposes.

For anaerobic bacteria, MICs were determined by the agar dilution method, in accordance with the guidelines of the CLSI (CLSI, 2007). The strains were defined as susceptible or resistant to the tested antibiotics according to the MIC breakpoints suggested by the CLSI (CLSI, 2007). Bacteroides fragilis ATCC 25285 was used as a reference strain for quality control.

Gram-negative aerobic bacteria and penicillin-resistant anaerobic isolates were tested for $\beta$-lactamase production using a chromogenic cephalosporin-based method (CLSI, 2007, 2009).

All experiments were repeated two or three times.

Statistical analysis. The differences in the number of bacteria recovered, and the number of resistant strains between odontogenic and non-odontogenic chronic sinusitis patients, were assessed with a $\chi^{2}$ test or Fisher's exact test, as appropriate. $P$-values $<0.05$ were considered significant.

\section{RESULTS}

\section{Odontogenic causes of chronic maxillary sinusitis}

Based on the symptoms and the instrumental tests performed, $20.3 \%$ of the sinusitis cases (12 cases of 59) were odontogenic. All 12 cases (eight female and four male) were associated with one or more oro-antral communications, and in two of them the presence of sinus polyps was also observed. Oro-antral communications were related to sinus lift procedures (five patients), third molar extractions (three patients), improper endosseous implant placement (two patients), excision of cysts (one patient) and presence of foreign bodies (fragments of teeth, one patient).

\section{Bacterial isolates}

Sixty aerobes and 75 anaerobes were recovered from the 47 cases of non-odontogenic sinusitis (2.9 bacteria per specimen); 15 aerobes and 25 anaerobes were isolated from the 12 patients with odontogenic sinusitis (3.3 bacteria per specimen) (Table 1). Mixed aerobic-anaerobic infections were found in 27 of $47(57 \%)$ cases of non-odontogenic sinusitis, and 9 of $12(75 \%)$ cases of odontogenic sinusitis. Aerobes and anaerobes alone were recovered in $9(19 \%)$ and 
Table 1. Number of bacterial isolates in non-odontogenic (47 patients) and odontogenic (12 patients) chronic sinusitis

\begin{tabular}{|c|c|c|}
\hline Bacteria & $\begin{array}{c}\text { Non-odontogenic } \\
\text { sinusitis }\end{array}$ & $\begin{array}{c}\text { Odontogenic } \\
\text { sinusitis }\end{array}$ \\
\hline \multicolumn{3}{|l|}{ Aerobic } \\
\hline Staphylococcus aureus & 23 & 4 \\
\hline Streptococcus pneumoniae & 12 & 4 \\
\hline Escherichia coli & 7 & 3 \\
\hline Haemophilus influenzae & 7 & \\
\hline Moraxella catarrhalis & 5 & \\
\hline Streptococcus pyogenes & 3 & \\
\hline Klebsiella species ${ }^{\star}$ & 2 & 1 \\
\hline Pseudomonas aeruginosa & 1 & \\
\hline Acinetobacter baumannii & & 1 \\
\hline Citrobacter koseri & & 1 \\
\hline Streptococcus constellatus & & 1 \\
\hline Subtotal & 60 & 15 \\
\hline \multicolumn{3}{|l|}{ Anaerobic } \\
\hline Peptostreptococcus species $\dagger$ & 24 & 7 \\
\hline Prevotella speciesł & 22 & 8 \\
\hline Porphyromonas species $\$$ & 11 & 4 \\
\hline Fusobacterium nucleatum & 10 & 4 \\
\hline Propionibacterium acnes & 7 & 1 \\
\hline Bacteroides fragilis & 1 & 1 \\
\hline Subtotal & 75 & 25 \\
\hline Total & 135 & 40 \\
\hline
\end{tabular}

${ }^{\star}$ Non-odontogenic: K. oxytoca 1, K. pneumoniae 1 ; odontogenic: $K$. pneumoniae 1.

$\dagger$ Non-odontogenic: $P$. anaerobius $20, P$. prevotii 4; odontogenic: $P$. anaerobius $6, P$. prevotii 1 .

$\$$ Non-odontogenic: P. intermedia 11, P. buccae 4, P. melaninogenica 4, $P$. oralis 3; odontogenic: $P$. intermedia 4, P. melaninogenica $2, P$. buccae 1 , P. oralis 1 .

§Non-odontogenic: P. asaccharolytica 7, P. gingivalis 4; odontogenic: P. asaccharolytica 3, $P$. gingivalis 1 .

$11(23 \%)$ of the non-odontogenic sinusitis specimens, respectively, and in $0(0 \%)$ and $3(25 \%)$ of the odontogenic sinusitis specimens, respectively. In both non-odontogenic and odontogenic sinusitis, the predominant aerobes were Staphylococcus aureus (23 and 4, respectively) and Streptococcus pneumoniae (12 and 4, respectively), while the most frequent anaerobic isolates were Peptostreptococcus species (24 and 7, respectively) and Prevotella species (22 and 8 , respectively) (Table 1$). H$. influenzae and $M$. catarrhalis were absent in odontogenic sinusitis (Table 1). Regarding the more common aerobic bacteria, Staphylococcus aureus was recovered from $46 \%$ (27/59) of the patients ( $49 \%$ and $33 \%$ of the non-odontogenic and of the odontogenic sinusitis specimens, respectively) and represented $36 \%$ (27/75) of the aerobic bacteria isolated, while Streptococcus pneumoniae was recovered from $27 \%(16 / 59)$ of the patients $(26 \%$ and $33 \%$ of the non-odontogenic and of the odontogenic sinusitis specimens, respectively) and represented $21 \%(16 / 75)$ of the aerobic bacteria isolated. Anaerobic bacteria were isolated from $85 \%(50 / 59)$ of the patients, and, in particular, in $81 \%$ $(38 / 47)$ of the non-odontogenic and $100 \%(12 / 12)$ of the odontogenic sinusitis patients.

There were no significant differences in the number of bacteria recovered between odontogenic and non-odontogenic sinusitis specimens $(P>0.05)$.

\section{Antimicrobial resistance of the isolated bacteria}

Overall, $22 \%(6 / 27)$ of the Staphylococcus aureus isolates were oxacillin-resistant, and $48 \%(13 / 27)$ were erythromycin-resistant, while only $4 \%(1 / 27)$ were resistant to moxifloxacin or quinupristin-dalfopristin, and no isolate was resistant to vancomycin (Table 2).

Fifty-six per cent $(9 / 16)$ of the Streptococcus pneumoniae isolates were penicillin-resistant and $56 \%(9 / 16)$ were erythromycin-resistant (Table 2). In particular, $38 \%(6 / 16)$ of the Streptococcus pneumoniae isolates were resistant both to penicillin and to erythromycin. Only $6 \%(1 / 16)$ of the Streptococcus pneumoniae isolates were resistant to moxifloxacin, while no isolate was resistant to quinupristindalfopristin (Table 2).

Many of the Gram-negative isolates were ampicillinresistant, but almost all were susceptible to moxifloxacin and co-trimoxazole, and none were resistant to amoxicillin-clavulanate or cefotaxime (Table 3). All ampicillinresistant isolates were $\beta$-lactamase producers (Table 3 ).

Thirty-five of the 100 anaerobic isolates $(35 \%)$ were resistant to penicillin (Table 4). In particular, 27 of the 61 $(44 \%)$ anaerobic Gram-negative isolates were penicillinresistant, all for $\beta$-lactamase production (Table 4). All bacteria were susceptible to metronidazole, with the relevant exception of the eight Propionibacterium acnes isolates, which were all resistant (Table 4). The great majority of Peptostreptococcus species $(94 \%, 29 / 31)$, Prevotella species $(90 \%, 27 / 30)$ and Fusobacterium nucleatum $(93 \%, 13 / 14)$ isolates and all the isolates of the other species were susceptible to moxifloxacin (Table 4). The rate of resistance to clindamycin was low for Peptostreptococcus species (10\%, $3 / 31)$, Prevotella species $(13 \%, 4 / 30)$ and Porphyromonas species $(13 \%, 2 / 15)$; no isolate of the other species was resistant to this antibiotic (Table 4).

The differences in the number of resistant strains between odontogenic and non-odontogenic sinusitis specimens were not significant $(P>0.05)$.

\section{DISCUSSION}

The close anatomical relationship of the maxillary sinus with the dental region means that dental infections and other odontogenic diseases can affect the maxillary sinus. Odontogenic infections that may involve the maxillary sinus include mainly acute and chronic periapical diseases, periodontal diseases and poorly performed endodontic treatments. Infection and sinusitis may also result from 
Table 2. In vitro activity of antimicrobial agents against aerobic Gram-positive isolates

\begin{tabular}{|c|c|c|c|c|c|c|}
\hline \multirow[t]{2}{*}{ Bacteria } & \multirow[t]{2}{*}{ No. isolates ${ }^{\star}$} & \multirow[t]{2}{*}{ Antimicrobial agent } & \multirow[t]{2}{*}{ No. resistant isolates ${ }^{\star}$} & \multicolumn{3}{|c|}{$\operatorname{MIC}\left(\mathrm{mg} \mathrm{l}^{-1}\right)$} \\
\hline & & & & $\mathrm{MIC}_{50}$ & $\mathrm{MIC}_{90}$ & MIC range \\
\hline \multirow[t]{7}{*}{ Staphylococcus aureus } & \multirow[t]{7}{*}{$27(23 / 4)$} & Penicillin & $20(18 / 2)$ & 1 & 8 & $0.06->32$ \\
\hline & & Oxacillin & $6(6 / 0)$ & 0.25 & 4 & $0.03-16$ \\
\hline & & Vancomycin & 0 & 0.25 & 1 & $\leqslant 0.015-2$ \\
\hline & & Erythromycin & $13(11 / 2)$ & 0.5 & 16 & $0.03->32$ \\
\hline & & Clindamycin & $9(9 / 0)$ & 0.25 & 16 & $0.03->32$ \\
\hline & & Quinopristin-dalfopristin & $1(1 / 0)$ & 0.25 & 0.5 & $\leqslant 0.015-4$ \\
\hline & & Moxifloxacin & $1(1 / 0)$ & 0.12 & 0.25 & $\leqslant 0.015-4$ \\
\hline \multirow[t]{5}{*}{ Streptococcus pneumoniae } & \multirow[t]{5}{*}{$16(12 / 4)$} & Penicillin & $9(6 / 3)$ & 2 & $>32$ & $\leqslant 0.015->32$ \\
\hline & & Erythromycin & $9(6 / 3)$ & 1 & $>32$ & $\leqslant 0.015->32$ \\
\hline & & Clindamycin & $7(5 / 2)$ & 0.25 & 32 & $\leqslant 0.015->32$ \\
\hline & & Quinopristin-dalfopristin & 0 & 0.12 & 0.5 & $\leqslant 0.015-1$ \\
\hline & & Moxifloxacin & $1(1 / 0)$ & 0.12 & 1 & $\leqslant 0.015-4$ \\
\hline \multirow[t]{4}{*}{ Streptococcus pyogenes } & \multirow[t]{4}{*}{$3(3 / 0)$} & Penicillin & 0 & - & - & $\leqslant 0.015-0.03$ \\
\hline & & Erythromycin & $1(1 / 0)$ & - & - & $0.06-8$ \\
\hline & & Clindamycin & 0 & - & - & $\leqslant 0.015-0.12$ \\
\hline & & Quinopristin-dalfopristin & 0 & - & - & $\leqslant 0.015-0.03$ \\
\hline
\end{tabular}

${ }^{\star}$ The numbers within parentheses indicate the number of isolates from patients with non-odontogenic/odontogenic sinusitis.

Table 3. In vitro activity of antimicrobial agents against aerobic Gram-negative isolates

\begin{tabular}{|c|c|c|c|c|c|c|}
\hline \multirow[t]{2}{*}{ Bacteria } & \multirow[t]{2}{*}{ No. isolates ${ }^{*}$} & \multirow[t]{2}{*}{ Antimicrobial agent } & \multirow[t]{2}{*}{ No. resistant isolates ${ }^{\star}$} & \multicolumn{3}{|c|}{$\operatorname{MIC}\left(\mathrm{mg} \mathrm{l}^{-1}\right)$} \\
\hline & & & & $\mathrm{MIC}_{50}$ & $\mathrm{MIC}_{90}$ & MIC range \\
\hline \multirow[t]{6}{*}{ Escherichia coli } & $10(7 / 3)$ & Ampicillin $\dagger$ & $4(4 / 0)$ & 8 & 32 & $\leqslant 0.03->64$ \\
\hline & & Amoxicillin-clavulanate & 0 & 2 & 4 & $\leqslant 0.03-4$ \\
\hline & & Cefotaxime & 0 & 1 & 2 & $\leqslant 0.03-4$ \\
\hline & & Ceftazidime & 0 & 0.12 & 1 & $\leqslant 0.03-2$ \\
\hline & & Moxifloxacin & $1(1 / 0)$ & 0.12 & 0.5 & $\leqslant 0.03-2$ \\
\hline & & Co-trimoxazole & $2(2 / 0)$ & 0.5 & 8 & $0.12-32$ \\
\hline \multirow[t]{6}{*}{ Klebsiella species } & $3(2 / 1)$ & Ampicillin $\dagger$ & $3(2 / 1)$ & - & - & $32->64$ \\
\hline & & Amoxicillin-clavulanate & 0 & - & - & $0.25-4$ \\
\hline & & Cefotaxime & 0 & - & - & $\leqslant 0.03-0.5$ \\
\hline & & Ceftazidime & 0 & - & - & $\leqslant 0.03-0.25$ \\
\hline & & Moxifloxacin & 0 & - & - & $0.06-0.25$ \\
\hline & & Co-trimoxazole & 0 & - & - & $0.5-2$ \\
\hline \multirow[t]{5}{*}{ Haemophilus influenzae } & $7(7 / 0)$ & Ampicillin $\dagger$ & $2(2 / 0)$ & 0.5 & - & $\leqslant 0.03-32$ \\
\hline & & Amoxicillin-clavulanate & 0 & 0.12 & - & $\leqslant 0.03-2$ \\
\hline & & Cefotaxime & 0 & 0.25 & - & $\leqslant 0.03-1$ \\
\hline & & Moxifloxacin & 0 & 0.25 & - & $\leqslant 0.03-0.5$ \\
\hline & & Co-trimoxazole & 0 & 0.5 & - & $0.06-2$ \\
\hline \multirow[t]{5}{*}{ Moraxella catarrhalis } & $5(5 / 0)$ & Ampicillin $\dagger$ & $5(5 / 0)$ & 32 & - & $16->64$ \\
\hline & & Amoxicillin-clavulanate & 0 & 0.12 & - & $\leqslant 0.03-1$ \\
\hline & & Cefotaxime & 0 & 0.06 & - & $\leqslant 0.03-0.5$ \\
\hline & & Moxifloxacin & 0 & 0.06 & - & $\leqslant 0.03-0.5$ \\
\hline & & Co-trimoxazole & 0 & 0.25 & - & $0.12-1$ \\
\hline
\end{tabular}

${ }^{\star}$ The numbers within parentheses indicate the number of isolates from patients with non-odontogenic/odontogenic sinusitis. $\dagger$ All the ampicillin-resistant strains were also $\beta$-lactamase-positive. 
Table 4. In vitro activity of antimicrobial agents against anaerobic isolates

\begin{tabular}{|c|c|c|c|c|c|c|}
\hline \multirow[t]{2}{*}{ Bacteria } & \multirow[t]{2}{*}{ No. isolates ${ }^{\star}$} & \multirow[t]{2}{*}{ Antimicrobial agent } & \multirow[t]{2}{*}{ No. resistant isolates ${ }^{\star}$} & \multicolumn{3}{|c|}{$\operatorname{MIC}\left(\mathrm{mg} \mathrm{l}^{-1}\right)$} \\
\hline & & & & $\mathrm{MIC}_{50}$ & $\mathrm{MIC}_{90}$ & MIC range \\
\hline \multirow{3}{*}{ Peptostreptococcus species } & & Clindamycin & $3(2 / 1)$ & 1 & 4 & $\leqslant 0.03-16$ \\
\hline & & Metronidazole & 0 & 1 & 4 & $\leqslant 0.03-8$ \\
\hline & & Moxifloxacin & $2(1 / 1)$ & 0.5 & 2 & $\leqslant 0.03-16$ \\
\hline \multirow{3}{*}{ Propionibacterium acnes } & & Clindamycin & 0 & 0.12 & - & $\leqslant 0.03-1$ \\
\hline & & Metronidazole & $8(7 / 1)$ & $>64$ & - & $>64$ \\
\hline & & Moxifloxacin & 0 & 0.12 & - & $\leqslant 0.03-1$ \\
\hline \multirow[t]{3}{*}{ Prevotella species } & $30(22 / 8)$ & Penicillin & $11(8 / 3) \dagger$ & 0.25 & $>64$ & $\leqslant 0.03->64$ \\
\hline & & Clindamycin & $4(4 / 0)$ & 0.5 & 8 & $\leqslant 0.03-32$ \\
\hline & & Metronidazole & 0 & 0.5 & 4 & $\leqslant 0.03-8$ \\
\hline Porphyromonas species & & Moxifloxacin & 0 & 0.25 & 1 & $\leqslant 0.03-2$ \\
\hline \multirow[t]{4}{*}{ Fusobacterium nucleatum } & $14(10 / 4)$ & Penicillin & $6(4 / 2) \dagger$ & 0.5 & 32 & $0.06-64$ \\
\hline & & Clindamycin & 0 & 0.12 & 1 & $\leqslant 0.03-1$ \\
\hline & & Metronidazole & 0 & 0.12 & 1 & $\leqslant 0.03-1$ \\
\hline & & Moxifloxacin & $1(0 / 1)$ & 0.12 & 2 & $\leqslant 0.03-8$ \\
\hline \multirow[t]{4}{*}{ Bacteroides fragilis } & $2(1 / 1)$ & Penicillin & $2(1 / 1) \dagger$ & - & - & $8-16$ \\
\hline & & Clindamycin & 0 & - & - & $0.12-0.5$ \\
\hline & & Metronidazole & 0 & - & - & 0.5 \\
\hline & & Moxifloxacin & 0 & - & - & $0.06-0.12$ \\
\hline
\end{tabular}

${ }^{\star}$ The numbers within parentheses indicate the number of isolates from patients with non-odontogenic/odontogenic sinusitis.

$\dagger \beta$-Lactamase-positive strains.

trauma to the dentition or from surgery in the posterior maxilla, including removal of teeth, alveolectomy, tuberosity reduction or other invasive surgical procedures that cause communications between the oral cavity and the maxillary sinus, such as maxillary sinus augmentation and implantology (Mehra \& Jeong, 2009).

It is reported that $5-40 \%$ of sinusitis cases occur through a dental cause (Björk, 1954; Maloney \& Doku, 1968; Melén et al., 1986), but the true incidence is difficult to determine accurately, and, as far as we know, in the last 20 years the incidence of a dental origin in chronic maxillary sinusitis has been rarely evaluated (Ugincius et al., 2006). In our study, $20.3 \%$ of the chronic maxillary sinusitis cases were of dental origin.

A recent meta-analysis (Arias-Irimia et al., 2010) evaluated the frequency of the different odontogenic conditions that may lead to maxillary sinusitis, which most commonly manifests itself as chronic maxillary sinusitis. According to the findings, iatrogenic conditions, and in particular tooth extractions, are more frequent than other aetiological factors, such as chronic periodontitis, although some authors (Melén et al., 1986; Nishimura \& Iizuka, 2002; Nimigean et al., 2006) have considered periodontal disease to be the most common way of spreading oral pathogens to the maxillary sinus. In our study, the causes of odontogenic sinusitis were always iatrogenic, and sinus lift procedures were the main aetiological factor, followed by tooth extractions.

Our microbiological findings showed that all specimens from chronic maxillary sinusitis were polymicrobial, and anaerobes were present in the great majority of specimens. According to Brook (2006), Gram-negative anaerobic bacteria, Peptostreptococcus species, Staphylococcus aureus and members of the Enterobacteriaceae were the commonest bacteria isolated, while $H$. influenzae and $M$. catarrhalis, which historically have an important role in the aetiology of acute sinusitis, were isolated with lower frequency. In contrast, we observed a higher frequency of Streptococcus pneumoniae, which in this study was recovered more frequently than was recently reported (Merino et al., 2003).

The microbiology of maxillary sinusitis associated with odontogenic infection is still little known. Data from the literature (Brook, 2005) showed that the most common organisms are anaerobic Gram-negative bacilli, Peptostreptococcus species, viridans streptococci and Staphylococcus aureus. Our results confirmed the high frequency of anaerobic bacteria and Staphylococcus aureus, but not of viridans streptococci. Moreover, in our study, 
comparing the microbiological findings of odontogenic with non-odontogenic sinusitis, a higher rate of recovery of Gram-negative anaerobes in odontogenic (43\%, 5/12 patients) than in non-odontogenic (32\%, 15/47 patients) sinusitis was noted.

These data confirm that anaerobic bacteria predominate not only in dental diseases, such as periodontal or periapical abscesses, but also in chronic maxillary sinusitis, associated or not with a dental origin. Moreover, anaerobic bacteria deserve more attention in the aetiopathogenesis of chronic sinusitis, since the frequency of $\beta$-lactamase-producing Gram-negative anaerobes is very high (Brook, 2005). Indeed, in our study, the rate of isolation of $\beta$-lactamaseproducing Gram-negative anaerobes was $44 \%$.

We also evaluated the susceptibility of the pathogenic bacteria isolated to many of the most commonly used antibiotics. In a recent study (Brook et al., 2008), the rate of recovery of meticillin-resistant Staphylococcus aureus in patients with chronic maxillary sinusitis was $12 \%$, while in our study, in keeping with the findings of Manarey et al. (2004), the rate was lower than $10 \%$.

The choice of antimicrobial treatment should be guided by properly collected bacterial culture results and local antibiotic resistance patterns. The frequent recovery of anaerobes, often $\beta$-lactamase-positive, and of meticillinresistant Staphylococcus aureus in chronic maxillary sinusitis suggests the use of antimicrobial agents effective against these organisms and with adequate sinus and oral bacteria coverage. The results of this study confirm that moxifloxacin, as previously reported (Milazzo et al., 2002; Speciale et al., 2002), has a good antibacterial activity against anaerobic pathogens and a wide activity against Grampositive and Gram-negative aerobes.

Many clinical studies have shown the efficacy of moxifloxacin in the treatment of acute maxillary sinusitis (Arrieta et al., 2007; Gehanno et al., 2003; Johnson et al., 2008; Rakkar et al., 2001; Zhou et al., 2010). In our opinion, the microbiological results of this study suggest that clinical trials should be designed to investigate the clinical usefulness of moxifloxacin in the treatment of chronic maxillary sinusitis also.

\section{REFERENCES}

Aral, M., Keles, E. \& Kaygusuz, I. (2003). The microbiology of ethmoid and maxillary sinuses in patients with chronic sinusitis. Am J Otolaryngol 24, 163-168.

Arias-Irimia, O., Barona-Dorado, C., Santos-Marino, J. A., MartínezRodriguez, N. \& Martínez-González, J. M. (2010). Meta-analysis of the etiology of odontogenic maxillary sinusitis. Med Oral Patol Oral Cir Bucal 15, e70-e73.

Arrieta, J. R., Galgano, A. S., Sakano, E., Fonseca, X., AmábileCuevas, C. F., Hernández-Oliva, G., Vivar, R., González, G., Torres, A. $\&$ the Moxifloxacin in Acute Sinusitis Study (MASS) Latin American Study Group (2007). Moxifloxacin vs amoxicillin/clavulanate in the treatment of acute sinusitis. Am J Otolaryngol 28, 78-82.
Benninger, M. S., Sedory Holzer, S. E. \& Lau, J. (2000). Diagnosis and treatment of uncomplicated acute bacterial rhinosinusitis: summary of the Agency for Health Care Policy and Research evidence-based report. Otolaryngol Head Neck Surg 122, 1-7.

Björk, H. (1954). Dental sinusitis; frequency and treatment. Acta Otolaryngol Suppl 43 (s118), 45-53.

Blandino, G., Milazzo, I., Fazio, D., Puglisi, S., Pisano, M., Speciale, A. \& Pappalardo, S. (2007). Antimicrobial susceptibility and betalactamase production of anaerobic and aerobic bacteria isolated from pus specimens from orofacial infections. J Chemother 19, 495-499.

Brook, I. (2005). Microbiology of acute and chronic maxillary sinusitis associated with an odontogenic origin. Laryngoscope 115, 823-825.

Brook, I. (2006). Bacteriology of chronic sinusitis and acute exacerbation of chronic sinusitis. Arch Otolaryngol Head Neck Surg 132, 1099-1101.

Brook, I., Foote, P. A. \& Hausfeld, J. N. (2008). Increase in the frequency of recovery of meticillin-resistant Staphylococcus aureus in acute and chronic maxillary sinusitis. J Med Microbiol 57, 1015-1017.

CLSI (2007). Methods for Antimicrobial Susceptibility Testing of Anaerobic Bacteria; Approved Standard, 7th edn. M11-A7 (vol. 27, no. 2). Wayne, PA: Clinical and Laboratory Standards Institute.

CLSI (2009). Methods for Dilution Antimicrobial Susceptibility Tests for Bacteria that Grow Aerobically; Approved Standard, 8th edn. M07-A8 (vol. 29, no. 2). Wayne, PA: Clinical and Laboratory Standards Institute.

CLSI (2010a). Methods for Antimicrobial Dilution and Disk Susceptibility Testing of Infrequently Isolated or Fastidious Bacteria; Approved Guideline, 2nd edn. M45-A2 (vol. 30, no. 18). Wayne, PA: Clinical and Laboratory Standards Institute.

CLSI (2010b). Performance Standards for Antimicrobial Susceptibility Testing, 20th Informational Supplement. M100-S20 (vol. 30, no. 1). Wayne, PA: Clinical and Laboratory Standards Institute.

Finegold, S. M., Flynn, M. J., Rose, F. V., Jousimies-Somer, H., Jakielaszek, C., McTeague, M., Wexler, H. M., Berkowitz, E. \& Wynne, B. (2002). Bacteriologic findings associated with chronic bacterial maxillary sinusitis in adults. Clin Infect Dis 35, 428-433.

Gehanno, P., Berche, P. \& Perrin, A. (2003). Moxifloxacin in the treatment of acute maxillary sinusitis after first-line treatment failure and acute sinusitis with high risk of complications. J Int Med Res 31, 434-447.

Johnson, P., Adelglass, J., Rankin, B., Sterling, R., Keating, K., Benson, A. \& Pertel, P. (2008). Acute bacterial maxillary sinusitis: time to symptom resolution and return to normal activities with moxifloxacin. Int J Clin Pract 62, 1366-1372.

Kremer, B., Jacobs, J. A., Soudijn, E. R. \& van der Ven, A. J. (2001). Clinical value of bacteriological examinations of nasal and paranasal mucosa in patients with chronic sinusitis. Eur Arch Otorhinolaryngol 258, 220-225.

Kretzschmar, D. P. \& Kretzschmar, J. L. (2003). Rhinosinusitis: review from a dental perspective. Oral Surg Oral Med Oral Pathol Oral Radiol Endod 96, 128-135.

Maloney, P. L. \& Doku, H. C. (1968). Maxillary sinusitis of odontogenic origin. J Can Dent Assoc (Tor) 34, 591-603.

Manarey, C. R., Anand, V. K. \& Huang, C. (2004). Incidence of methicillin-resistant Staphylococcus aureus causing chronic rhinosinusitis. Laryngoscope 114, 939-941.

Mehra, P. \& Jeong, D. (2009). Maxillary sinusitis of odontogenic origin. Curr Allergy Asthma Rep 9, 238-243.

Melén, I., Lindahl, L., Andréasson, L. \& Rundcrantz, H. (1986). Chronic maxillary sinusitis. Definition, diagnosis and relation to 
dental infections and nasal polyposis. Acta Otolaryngol 101, 320-327.

Merino, L. A., Ronconi, M. C., Hreñuk, G. E. \& de Pepe, M. G. (2003). Bacteriologic findings in patients with chronic sinusitis. Ear Nose Throat J 82, 798-800, 803-804, 806.

Milazzo, I., Blandino, G., Musumeci, R., Nicoletti, G., Lo Bue, A. M. \& Speciale, A. (2002). Antibacterial activity of moxifloxacin against periodontal anaerobic pathogens involved in systemic infections. Int $J$ Antimicrob Agents 20, 451-456.

Murray, P. R., Baron, E. J., Jorgensen, J. H., Landry, M. L. \& Pfaller, M. A. (2007). Manual of Clinical Microbiology, 9th edn. Washington, DC: American Society for Microbiology.

Niederfuhr, A., Kirsche, H., Riechelmann, H. \& Wellinghausen, N. (2009). The bacteriology of chronic rhinosinusitis with and without nasal polyps. Arch Otolaryngol Head Neck Surg 135, 131-136.

Nimigean, V. R., Nimigean, V., Maru, N., Andressakis, D., Balatsouras, D. G. \& Danielidis, V. (2006). The maxillary sinus and its endodontic implications: clinical study and review. B-ENT 2, $167-175$.
Nishimura, T. \& lizuka, T. (2002). Evaluation of the pathophysiology of odontogenic maxillary sinusitis using bone scintigraphy. Int J Oral Maxillofac Surg 31, 389-396.

Rakkar, S., Roberts, K., Towe, B. F., Flores, S. M., Heyd, A. \& Warner, J. (2001). Moxifloxacin versus amoxicillin clavulanate in the treatment of acute maxillary sinusitis: a primary care experience. Int J Clin Pract 55, 309-315.

Speciale, A., Musumeci, R., Blandino, G., Milazzo, I., Caccamo, F. \& Nicoletti, G. (2002). Minimal inhibitory concentrations and time-kill determination of moxifloxacin against aerobic and anaerobic isolates. Int J Antimicrob Agents 19, 111-118.

Summanen, P., Baron, E. J., Citron, D. M., Strong, C. A., Wexler, H. M. \& Finegold, S. M. (1993). Wadsworth Anaerobic Bacteriology Manual, 5th edn. Belmont, CA: Star Publishing.

Ugincius, P., Kubilius, R., Gervickas, A. \& Vaitkus, S. (2006). Chronic odontogenic maxillary sinusitis. Stomatologija 8, 44-48.

Zhou, B., Jiang, X., Zhai, L., Xiao, S., Wang, J., Xiao, G., Ruan, B., Liang, C., Ye, Q. \& other authors (2010). Moxifloxacin in the treatment of acute bacterial rhinosinusitis: results of a multicenter, non-interventional study. Acta Otolaryngol 130, 1058-1064. 